\title{
Immune system of the inner ear as a novel therapeutic target for sensorineural hearing loss
}

\author{
Takayuki Okano* \\ Department of Otolaryngology, Head and Neck Surgery, Graduate School of Medicine, Kyoto University, Kyoto, Japan
}

\section{Edited by: \\ Hiroshi Hibino, Niigata University, Japan}

\section{Reviewed by:}

Fumiaki Nin, Niigata University, Japan Wen-Jie Song, Kumamoto University

School of Medicine, Japan

*Correspondence:

Takayuki Okano, Department of Otolaryngology, Head and Neck

Surgery, Graduate School of

Medicine, Kyoto University,

54 Shogoin Kawaharacho, Sakyo,

Kyoto 606-8507, Japan

e-mail: tokano@ent.kuhp.kyoto-u.ac.jp

\begin{abstract}
Sensorineural hearing loss ( $\mathrm{SNHL}$ ) is a common clinical condition resulting from dysfunction in one or more parts in the auditory pathway between the inner ear and auditory cortex. Despite the prevalence of $\mathrm{SNHL}$, little is known about its etiopathology, although several mechanisms have been postulated including ischemia, viral infection or reactivation, and microtrauma. Immune-mediated inner ear disease has been introduced and accepted as one SNHL pathophysiology; it responds to immunosuppressive therapy and is one of the few reversible forms of bilateral SNHL. The concept of immune-mediated inner ear disease is straightforward and comprehensible, but criteria for clinical diagnosis and the precise mechanism of hearing loss have not been determined. Moreover, the therapeutic mechanisms of corticosteroids are unclear, leading to several misconceptions by both clinicians and investigators concerning corticosteroid therapy. This review addresses our current understanding of the immune system in the inner ear and its involvement in the pathophysiology in SNHL. Treatment of SNHL, including immune-mediated inner ear disorder, will be discussed with a focus on the immune mechanism and immunocompetent cells as therapeutic targets. Finally, possible interventions modulating the immune system in the inner ear to repair the tissue organization and improve hearing in patients with SNHL will be discussed. Tissue macrophages in the inner ear appear to be a potential target for modulating the immune response in the inner ear in the pathophysiology of SNHL.
\end{abstract}

Keywords: resident macrophages, autoimmunity, corticosteroids, cell therapy, molecular targeted drugs

\section{INTRODUCTION}

Sensorineural hearing loss (SNHL) is a collection of common auditory disorders resulting from dysfunction of the inner ear, auditory nerve, or the auditory processing pathway in the central nervous system. SNHL comprises a wide variety of auditory disorders including sudden deafness, age-related hearing loss, noise-induced hearing loss, and Meniere's disease. To date, very little of the SNHL pathophysiology is known because biopsy of the human inner ear is not feasible. Among the purposed mechanisms, immune-mediated SNHL has been introduced and accepted in the last three decades.

The inner ear has been thought of as an immune privileged organ for a long time. The cochlea has no lymphatic drainage, and the blood-labyrinth barrier is tightly controlled to separate the cochlear microenvironment from the circulation. In addition, the concentration of immunoglobulin in the cochlear fluid is $1 / 1,000$ of the concentration in the cerebrospinal fluid (Harris and Ryan, 1984). McCabe (1979) introduced the clinical definition of autoimmune inner ear disease as rapidly progressive bilateral hearing loss that responds to corticosteroid and immunosuppressive therapy. Corticosteroids have been widely used as the first and only regimen to treat virtually all types of SNHL with sudden onset or rapid progression even before McCabe's report. The anti-inflammatory and immune suppressive functions of corticosteroids led to their common use for hearing loss, especially when inflammation or an immunological disorder is suspected. Despite the common use of corticosteroids for inner ear disorders, our understanding of their role in the pathogenesis of reversible hearing loss remains limited. Steroid-responsive hearing loss does not always indicate an underlying inflammation or immune disorder in the inner ear (Trune and Canlon, 2012). Topical application of corticosteroids in the tympanic cavity has also been reported in patients unable to tolerate systemic treatment due to global adverse effects (Kakehata et al., 2006; Han et al., 2009), and the functional mechanisms of systemic and topical corticosteroid application supposedly differ. Therefore, a better understanding of the inner ear immunology and mechanisms of corticosteroids in the inner ear would enable development of a more sophisticated therapy for SNHL, including immune-mediated inner ear disease. In addition, alternative strategies of modulating immune activity without corticosteroids are desirable for treating certain types of SNHL.

In this review, we will discuss the characteristics and suspected pathophysiology of clinical hearing loss mediated by the immune system. Second, we will describe the current understanding of the inner ear immune system and will explore recent advances in both basic and clinical research of the mechanism of corticosteroid therapy in the inner ear. Finally, we will discuss current and potential SNHL therapies, including treatments targeting immune-mediated inner ear disease.

\section{PATHOPHYSIOLOGY OF SNHL FROM AN IMMUNOLOGICAL VIEWPOINT}

The pathophysiology of organ-specific autoimmune disease is believed to be initiated by three primary mechanisms: 
(i) production of autoantibodies against tissue antigens, (ii) deposition of antigen-antibody complexes in tissue, and (iii) infiltration and destruction of tissue by specific cytotoxic Tcells. To date, the mechanisms of hearing loss in immune-mediated inner ear disease has yet to be determined, and none of the three described pathophysiology mechanisms have been reported in the human inner ear.

Immune-mediated inner ear disease was originally defined by McCabe (1979), who stated that idiopathic bilateral SNHL progresses to deafness over weeks or months, not hours, days, or years, and responds to corticosteroid and immunosuppressive therapy. The term of autoimmune inner ear disease refers to a pathology restricted to the inner ear. The time course of hearing loss distinguishes immune-mediated inner ear disease from sudden deafness or age-related hearing loss. Although this clinical entity is probably immune-mediated as immunosuppressive agents are effective, there is no direct evidence that the condition is autoimmune in etiology because diagnostic biopsy of the human inner ear is not feasible. Moreover, there are no uniformly accepted diagnostic criteria of immune-mediated inner ear disease. The presence of bilateral SNHL of at least $30 \mathrm{~dB}$ with evidence of progression in at least one ear on two serial audiograms performed less than 3 months apart is often used as case criteria (Moscicki et al., 1994). Fluctuations in hearing may occur, and immune-mediated disease is one of the few reversible causes of SNHL. Further study is still required to determine the pathophysiologic mechanisms underlying immune-mediated inner ear disease.

The pathology of Meniere's disease has historically been defined as an inner ear disorder presenting with endolymphatic hydrops. It is well known that some patients with Meniere's disease show remarkable recovery from fluctuating and refractory SNHL or vertigo following systemic corticosteroid treatment; therefore, an immune-mediated mechanism has been implicated in the pathology of Meniere's disease (Hughes et al., 1983; Derebery etal., 1991). In a study of patients with Meniere's disease, immunohistochemistry showed IgG deposition in the endolymphatic sac obtained by surgical biopsy in 10 of 23 patients (Dornhoffer et al., 1993). Alleman reported that 3 of $30(10 \%)$ patients with Meniere's disease showed a positive serum reaction against proteins extracted from the endolymphatic sac of autopsy samples, and clinical data have shown an association between immunoreactivity and the disease severity (Alleman etal., 1997), suggesting an autoimmune component in some cases of Meniere's disease. In contrast, other studies report a relationship between herpes simplex virus and the pathology of Meniere's disease (Bergstrom et al., 1992; Kumagami, 1996). Although it appears likely that an immune reaction is involved in the pathophysiology of Meniere's disease, the mechanism of endolymphatic hydrops, whether caused by viral infection, autoimmune reaction, or both, remains to be elucidated.

Multisystemic, organ-nonspecific autoimmune pathology may involve the inner ear, leading to secondary SNHL. A limited number of studies have evaluated human temporal bones from patients with autoimmune disease, such as Wegener granulomatosis, polyarteritis nodosa, Cogan syndrome, and lupus (McCabe, 1989;
Moscicki et al., 1994). Some specimens showed fibrosis and osteoneogenesis, consistent with the end stage of inflammation. Other bones demonstrated atrophy of the stria vascularis, the organ of Corti, and the spiral ganglion without evidence of inflammation, findings consistent with ischemia. Dettmer et al. (2011) reported that the temporal bones of Crohn's disease patients with granulomatous inner ear disease demonstrated mild chronic inflammation, poorly defined granulomas, and infiltration of CD68-positive macrophages.

Cytomegalovirus (CMV) is the leading cause of human nonhereditary congenital hearing loss. Approximately 10-20\% of children with congenital CMV infection exhibit varying degrees of hearing loss (Barbi et al., 2003; Numazaki and Fujikawa, 2004). However, the pathology of congenital CMV infection within the inner ear is poorly understood. Various animal models have been employed to study the pathology of SNHL caused by intrauterine CMV infection (Woolf et al., 1989; Juanjuan et al., 2011; Wang et al., 2013). Two studies using mouse CMV infection models reported that CMV DNA was detected in spiral ganglion neurons and the stria vascularis (Juanjuan et al., 2011; Wang et al., 2013), suggesting a potential therapeutic target in CMVinduced SNHL. Multiple studies have focused on developing effective vaccines or antiviral therapy for congenital CMV infection. Unfortunately, there is no clinically effective vaccine for congenital CMV infection or CMV-induced SNHL (Arvin et al., 2004).

Several mechanisms have been postulated as the pathophysiology of sudden deafness, including microcirculatory disturbances caused by thrombosis, microtrauma or rupture of endolymph, viral infection or reactivation, and immune-mediated reaction.

One of the main pathophysiology theories of idiopathic sudden deafness is that viral infection or reactivation in the inner ear damages critical structures in the cochlea. Increased serum concentrations of antibodies against CMV, herpes zoster, herpes simplex type 1 , influenza $B$, and mumps have been reported in patients with idiopathic sudden deafness (Merchant et al., 2008; Pyykko and Zou, 2008). Cochlear enhancement on magnetic resonance imaging (MRI) is a potential sign of inflammation in the inner ear and has been observed in some patients suffering from sudden deafness (Stokroos et al., 1998). The inner ear enhancement on MRI disappeared following resolution of hearing loss in 2 of 12 patients with sudden deafness (Mark etal., 1992). Garcia-Berrocal et al. (1997) reported a decreased concentration of both $\mathrm{CD}^{+}$and $\mathrm{CD}^{+}$cells in patients compared to healthy control subjects, suggesting an abnormal autoimmune response in lymphocyte subpopulations in patients with sudden deafness. In addition, western blot assay showed a response to recombinant human heat shock protein 70, a non-specific heat shock protein, in 19 of 58 (33\%) patients with idiopathic SNHL (Tebo et al., 2006). An analysis of 11 human temporal bones from patients with sudden SNHL showed that the morphology of the stria vascularis and spiral ligament were relatively preserved, supporting a viral etiology rather than a vascular insufficiency (Linthicum et al., 2013). These findings suggest that immune mechanisms, including $\mathrm{T}$ cell-mediated and antibody responses, are involved, at least in part, in the onset or progression of idiopathic sudden deafness. 


\section{EVIDENCE OF THE IMMUNE SYSTEM IN THE INNER EAR}

As previously mentioned, the inner ear was believed to be "immune-privileged" and to exclude all immunocompetent cells, except in the endolymphatic sac, for a long time because chronic degeneration without neutrophilic infiltration in the organ of Corti has been described in patients with presbycusis or hearing loss due to chronic noise exposure. However, Rask-Andersen and Stahle (1979) initiated a new era of inner ear immunology by describing intimate contact between the lymphocytes and macrophages in the endolymphatic sac of guinea pigs. This association suggested that two cell types mediated the antigen-presenting process in the endolymphatic sac. The presence of immunocompetent cells and phagocytized antigen within macrophages was also reported in the endolymphatic sac (Harris etal., 1997). These findings revealed the specific role of the endolymphatic sac in antigen processing and immune activity in the inner ear. However, recent studies have demonstrated the presence of immunoreactive cells in other areas of the inner ear even under normal conditions (Lang et al., 2006; Okano et al., 2008; Sato et al., 2008). Lang et al. (2006) reported that bone marrowderived cells of hematopoietic origin migrate into the cochlea and reside in the cochlear modiolus and the cochlear lateral wall. They also showed that bone marrow-derived cells in the cochlea express ion transporters such as the sodium/potassium/chloride co-transporter or sodium/potassium-ATPase in the cochlear lateral wall, which contains several types of fibrocytes. In a study using bone marrow-chimeric mice that were transplanted with hematopoietic stem cells after receiving lethal systemic irradiation, Okano et al. (2008) demonstrated that bone marrow-derived cells reside as macrophages in the cochlea. They also reported that Iba1-positive macrophages were continuously and slowly replaced by bone marrow-derived cells from the systemic circulation over several months. Finally, Sato et al. (2008) reported that bone marrow-derived cells expressing CX3CR1, a fractalkine receptor specific to monocytes, natural killer cells, activated T-cells, and tissue macrophages, reside in the spiral ganglion and spiral ligament. In addition, they showed that CX3CR1-positive cells were repopulated in the cochlea over several months. Collectively, these findings indicate that the inner ear harbors immunocompetent cells of hematopoietic origin normally, with most cells likely to be tissue macrophages phenotypically. Although these tissue macrophages are distributed primarily in the spiral ganglion, spiral limbus, and spiral ligament, macrophage-like melanocytes are also observed in the intermediate layer of the stria vascularis (Zhang et al., 2012). These melanocytes reside adjacent to blood vessels and are believed to be perivascular-resident macrophages that contribute to the formation of the blood-intrastrial fluid barrier (Figure 1).

The role of cochlear macrophages and mechanisms of macrophage migration into the cochlea remain largely unknown. Previous studies demonstrated that injury of the auditory sensory epithelium induces inflammation characterized by macrophage infiltration into the chick basilar papilla (Warchol, 1997; Bhave etal., 1998). A large increase in the number of CD45-positive cells has been observed after noise exposure in the mouse cochlea, suggesting inflammation caused by bone marrowderived cells (Hirose et al., 2005; Tornabene et al., 2006). The

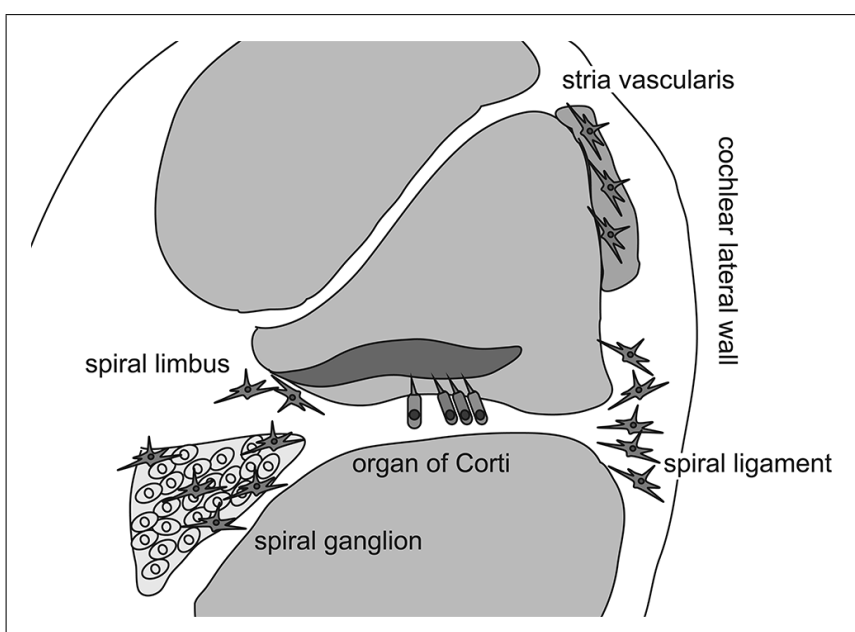

FIGURE 1 | Distribution of cochlear macrophages. Schematic drawing shows the cross section of the cochlear duct. Cochlear macrophages reside in the spiral ligament and spiral limbus where fibrocytes are located to keep ion exchanges. In addition, macrophages are distributed in the spiral ganglion. Finally, macrophage-like melanocytes are observed in the intermediate layer of the stria vascularis.

number of cochlear macrophages is also increased after aminoglycoside insult in both the spiral ganglion and spiral ligament (Sato etal., 2010). These findings indicate that cochlear macrophages play important roles in the onset and progression of inflammation after damage to the cochlear sensory epithelium. Macrophages in the cochlea are likely involved not only in the degeneration of the organ of Corti, but also the stria vascularis. Jabba et al. (2006) reported that hyperpigmentation of the stria vascularis and reorganization of marginal cells occurs in Slc26a4-null mice, a mouse model of Pendred syndrome, and is associated with the invasion of CD68-positive macrophages. Similar findings regarding hyperpigmentation of the stria vascularis have also been reported in genetically modified mice (Singh and Wangemann, 2008; Lu et al., 2012). The invasion of macrophages is restricted to the degenerated stria vascularis, suggesting contribution from the cochlear macrophages to degeneration or regeneration of the stria vascularis and the cochlear lateral wall.

The number of cochlear macrophages is also increased by systemic administration of macrophage colony stimulating factor (Csf1), which is one of the primary regulators of mononuclear phagocyte activation. The density of Ibal-positive macrophages is increased in both the spiral ligament and spiral ganglion 1 day after administering Csf1 (Okano etal., 2008), but it is unclear whether the increased macrophage population is due to migration from the circulation or in situ proliferation in the cochlea. Yagihashi et al. (2005) also demonstrated that topical administration of Csfl ameliorates the degradation of auditory neurons following surgical injury in a rat model. In addition, Csf1 was demonstrated to have neuroprotective properties in an in vitro model of excitotoxicity in hippocampal neurons, suggesting both direct and indirect effects of Csf1 on survival of targeted cells (Vincent et al., 2002). It is unknown whether the activation of tissue 
macrophages has protective or degenerative effects in the target organ, but control of macrophage activity through Csf1 administration is a potential approach for several inner ear disorders.

Previous reports investigating in situ proliferation of cochlear macrophages are controversial. Using Bromodeoxyuridine labeling, Hirose etal. (2005) reported that cochlear macrophages do not proliferate after acoustic trauma. However, according to the study done by Okano et al. (2008) a subset of macrophages expressed Ki67, suggesting that resident macrophages enter the cell cycle after migration following surgical invasion of the cochlea. Although the precise nature of migrating macrophages is to be determined, cochlear macrophages are most likely responsible for several different inner ear pathologies.

\section{TISSUE MACROPHAGES IN THE INNER EAR}

In general, adaptive immune cells play a major role in disease progression, and the innate immune system, primarily monocytes and macrophages, plays a central role in the onset of immune activity. The concept of multiple macrophage activation states is not new, but extending this idea to resident tissue macrophages has garnered increased interest in recent years. Unfortunately, research of peripheral macrophage polarization might not accurately describe their central nervous system counterparts.

Macrophages are derived from monocyte precursors that undergo tissue-specific differentiation and infiltrate the site of infection or injury to produce inflammatory mediators. The cells typically polarize into the pro-inflammatory M1 phenotype and function as an effector of the Th1-mediated immune response. The M1 polarization of macrophages is regulated by several factors including the mineralocorticoid receptor (Lawrence and Natoli, 2011). In the normal course of inflammation, the immune process is controlled, and M1-macrophages undergo apoptosis or switch to the anti-inflammatory M2 phenotype, thereby halting inflammation. However, if the inflammatory response of macrophages is not controlled, it becomes pathogenic, resulting in significant levels of non-specific tissue damage and leading to inflammatory and autoimmune diseases (Wynn et al., 2013). Therefore, macrophage-targeted therapy is extremely relevant in improving the prognosis of inflammatory diseases, particularly inflammation in the inner ear.

Thought provoking observations have been obtained in studies of patients with human immunodeficiency virus (HIV), specifically concerning macrophage function in the inner ear. Monocytes and macrophages are susceptible to HIV infection and are considered a main mechanism responsible for central nervous system infection in areas containing perivascular macrophages and parenchymal microglia (Burdo et al., 2013). Lin et al. (2013) demonstrated that HIV infection is significantly associated with an increased risk of developing sudden deafness in patients aged between 18 and 35 years. In addition, Assuiti et al. (2013) found no direct association between anti-retroviral therapy and hearing loss but stressed the need for future investigation of the causes and association between anti-retroviral therapy and hearing loss. These data suggest that deficiencies in the macrophage and monocyte lineage may lead to dysfunction in the inner ear and highlight the important roles of macrophages in the maintenance of auditory function.

Several surface markers have been used in the animal studies of macrophages to immunohistochemically test their phenotypes and distribution in the tissues. CD68 is a heavily glycosylated transmembrane protein and is a common surface marker expressed in all macrophages (Smith and Koch, 1987; Ramprasad et al., 1996). F4/80 is a member of a gene family that includes the human epidermal growth factor module-containing mucinlike hormone receptor 1 and human CD97, and resides on the surface of a family of cells that includes all well differentiated members of the mononuclear phagocyte system. Although the precise function of F4/80 is not completely understood as F4/80null mice have no remarkable phenotype, F4/80-positive cells have many common features regardless of their tissue location and are characterized by highly ramified cell shape (Hume et al., 2002). Iba1 is a calcium binding protein specific to macrophages that mediates calcium signals that may control migration and phagocytosis in tissue macrophages (Imai et al., 1996). Reportedly, tissue macrophages in the inner ear express Iba1 in addition to F4/80 (Okano etal., 2008). Csf1r is an alternative surface marker on macrophages and is thought to play key roles in the proliferation, differentiation, and survival of macrophages (Hume et al., 2002). In other categorical systems, the differentiation of monocytes and macrophages is described based on the expression of specific cell markers. If similar markers could be identified in tissue macrophages or cells of monocyte lineage, it may be possible to trace these cells along several different points of the inner ear pathophysiology, including systemically circulating monocytes, migrating monocytes, and resident tissue macrophages.

\section{CORTICOSTEROID THERAPY}

Systemic or possibly local administration of corticosteroids is the mainstay of treatment for SNHL, including immune-mediated inner ear disease. However, there are limited prospective data evaluating the appropriate dose, route, and length of corticosteroid treatment. In addition, although many patients experience a short-term response to steroids, the response is generally not sustained (Zeitoun etal., 2005). A prospective, randomized, controlled study in 116 patients with rapidly progressive, bilateral SNHL reported that $57 \%$ of patients in the 1 month prednisone challenge showed improved hearing, but adverse effects such as hyperglycemia were observed in 14\% of patients (Alexander etal., 2009). A meta-analysis of the management of idiopathic sudden SNHL performed by Spear and Schwartz (2011) reported that intratympanic corticosteroids administered as the primary treatment appeared equivalent to treatment with high-dose oral prednisone. Furthermore, intratympanic administration of corticosteroids potentially recovered some degree of hearing as a salvage therapy. These observations suggest that the local administration of corticosteroids is beneficial through mechanisms distinct from those of systemic corticosteroid therapy.

Despite numerous clinical reports of corticosteroid treatment for SNHL, the spontaneous rate of recovery in acute SNHL complicates conclusions about corticosteroid efficacy. To date, 
the mechanisms underlying fluctuating SNHL in an immunemediated inner ear disease are unclear. We know little on how corticosteroids work in the inner ear and which parts of the inner ear are affected during reversible hearing loss. The expression of glucocorticoid receptors in the inner ear is limited to the inner and outer hair cells, the spiral ganglion, and the spiral ligament (Tahera et al., 2006; Meltser et al., 2009). In addition to glucocorticoid receptors, corticosteroids have a strong affinity for mineralocorticoid receptors. The use of systemic mineralocorticoids alone or in combination with glucocorticoids has not been evaluated in humans, but is apparently efficacious in animal models (MacArthur etal., 2008). Because the inner ear requires tight regulation of ion homeostasis in both the perilymph and endolymph, the effect of corticosteroid therapy through mineralocorticoid receptors should be considered in the mechanism of action when treating SNHL. Moreover, there are several questions on the assumptions which clinicians and researchers take for granted. Do corticosteroids only suppress inflammation and immune response in the inner ear? Do corticosteroids affect the inner ear specifically or do the systemic effects of corticosteroids benefit the inner ear disorder? Does immune-mediated hearing loss always respond to corticosteroids? A better understanding of the immune-mediated aspects of hearing loss and specific diagnostic assays would lead to the development of immune-modulating therapy for sudden or progressive SNHL.

McCabe recommended high-dose corticosteroids along with cyclophosphamide therapy for prolonged treatment of immunemediated inner ear disease (McCabe, 1979). However, the extended follow-up of patients treated with cyclophosphamide revealed potential adverse effects and long-term morbidity and mortality risks of the agent, particularly neoplasm development in younger patients, which limited its use and prompted the search for other immunosuppressive options (Harris et al., 2003; Garcia-Berrocal et al., 2006).

Methotrexate has been used as a sparing treatment to control refractory immune-mediated SNHL. Salley et al. (2001) reported improvement in the majority of 53 patients with immune-mediated inner ear diseases who were treated with low-dose methotrexate. Long-term, low-dose methotrexate therapy appeared to be effective in at least some patients with immune-mediated hearing loss that is refractory to traditional corticosteroid therapy (Matteson et al., 2001). By contrast, a randomized, double-blind, placebo-controlled trial in 2003 of immune-mediated inner ear disease suggested that methotrexate does not appear to be effective in maintaining the hearing improvement achieved with prednisone therapy (Harris et al., 2003).

Azathioprine was also reported as an alternative option in treating immune-mediated inner ear disease, although reports were based on small case series and were inconclusive (Lasak et al., 2001).

According to these findings, systemic immunosuppressives such as methotrexate are effective in some patients with bilateral, progressive, or fluctuating SNHL, which indicates an immune component in the pathophysiology of hearing loss. However, the diagnostic criteria of immune-mediated inner ear disease vary among previous reports. Clinicians and investigators should consider that patients with bilateral fluctuating SNHL do not always have an immune disorder in the inner ear.

\section{RECENT ADVANCES AND FUTURE DIRECTIONS OF SNHL TREATMENT \\ MOLECULAR-TARGETED DRUGS AND BIOLOGICAL AGENTS}

Despite initial optimistic reports suggesting a therapeutic effect of methotrexate, a recent study by Harris et al. (2003) failed to demonstrate its efficacy for long-term management of immune-mediated inner ear diseases as mentioned above. Instead, molecular-targeted drugs have garnered attention of investigators and clinicians in the fields of immunology and audiology due to their specificity against therapeutic targets, resulting in less toxicity and fewer adverse effects.

Etanercept is a fusion protein comprising two recombinant tumor necrosis factor (TNF) receptors linked to the $\mathrm{C}$ portion of human IgG1 (Mohler etal., 1993). A retrospective case series by Rahman etal. (2001) examined the response to etanercept in 12 patients with immune-mediated haring loss responsive to high-doses of corticosteroids. Improvement or stabilization of hearing and tinnitus was observed in $91 \%$ of patients, suggesting that etanercept therapy is safe and may be efficacious in some patients with immune-mediated hearing loss. By contrast, two studies reported that etanercept has no substantial efficacy in improving hearing loss (Cohen et al., 2005; Matteson et al., 2005). Further studies are needed evaluating alternative regimens that use etanercept or other anti-TNF-alpha agents.

Infliximab is another monoclonal antibody against TNFalpha that binds TNF-alpha and reduces its activity (Siddiqui and Scott, 2005). A retrospective review of eight patients with suspected immune-mediated hearing loss refractory to conventional treatment examined the efficacy of infliximab on hearing improvement; however, none of the patients exhibited a positive response to infliximab therapy based on objective measurements (Liu etal., 2011). Monoclonal antibody therapy directly targeting cells in the inner ear is unlikely to be effective because the concentration of immunoglobulin is much lower in this region than that in cerebrospinal fluid or blood due to tight regulation by the blood-labyrinthine barrier. Accordingly, transtympanic administration of infliximab was evaluated by Van Wijk etal. (2006) in nine patients with immune-mediated hearing loss. Transtympanic administration of infliximab resulted in hearing improvement and reduced disease relapses, indicating the potential utility of local administration of monoclonal antibody in treating inner ear disease.

Adalimumab was also used to block TNF signaling in patients with immune-mediated hearing loss, but reports were based on a small number of cases (Morovic Vergles et al., 2010).

Rituximab is a genetically engineered chimeric monoclonal antibody against CD20, which resides the surface of B cells. The agent reduces autoantibody production both in circulating and tissue B cells, but does not affect plasma cells. A small 
pilot study in patients with immune-mediated inner ear diseases was performed evaluating the efficacy of rituximab in treating hearing loss (Cohen et al., 2011). Further evaluation of rituximab is encouraged using a properly designed randomized study.

\section{NUCLEIC ACID THERAPY}

Nucleic acid therapy, including delivery of gene constructs to increase or force expression in the targeted tissue, and small interfering RNA to block expression of a specific gene, is a promising approach for treating inner ear disease. However, limited access to the lesion site creates challenges in nucleic acid therapy of the inner ear. Various studies employing animal models utilize viral vectors to introduce the nucleic acid into the inner ear, but there are toxicity and safety concerns associated with this method, including immunogenicity and mutagenesis. Non-viral vectors are advantaged by overcoming these limitations plaguing viral vectors. Although nucleic acid therapy is challenging in the in vivo setting, the development of novel delivery systems could lead to drastic advances in improving the prognosis of patients with SNHL. Obviously, macrophages are a potential target for nucleic acid therapy using novel delivery systems in the inner ear, controlling not only inflammation and degeneration of sensory organs, but also regeneration of the cochlear lateral wall and innervation from the spiral ganglion neurons to hair cells.

\section{DELIVERY OF GENE MODIFIED MACROPHAGES}

The last, but not least, the use of genetically modified monocytes or macrophages as vectors should be considered for production of therapeutic molecules or factors that promote regeneration or regrowth of specific structures in the inner ear. This concept is especially well suited for a secreted paracrine or endocrine factor such as a hormone or growth factor. Because the inner ear contains three fluid-filled compartments, secreted factors from genetically modified macrophages could potentially diffuse throughout the inner ear without help from the blood or lymphatic circulation. Although the use of genetically modified cells as vectors of genes or pharmacotherapeutic reagents is in the early stage (Hakuba et al., 2005; Okano et al., 2006; Kesser and Lalwani, 2009), transplantation of genetically engineered cells able to secrete specific metabolic or humoral cues could augment pharmacologic immune modulation in the inner ear. Delivery of genetically modified cells into the inner ear could pose a major challenge because of the anatomical characteristics of the inner ear. Monocytes and macrophages are able to migrate into the inner ear in both pathologic and normal conditions (Hirose et al., 2005; Okano et al., 2008). Thus, the human monocyte lineage could be isolated and cultured ex vivo and genetically manipulated. Intravenous administration of genetically modified monocytes could enable them to reach and migrate into the inner ear, although tissue- or organ specificity could be a potential problem to overcome in clinical applications (Figure 2).

Apart from resident macrophages at the disease site, circulating monocytes are continuously recruited to meet the demands of the inflammatory response and the expression of chemokines,

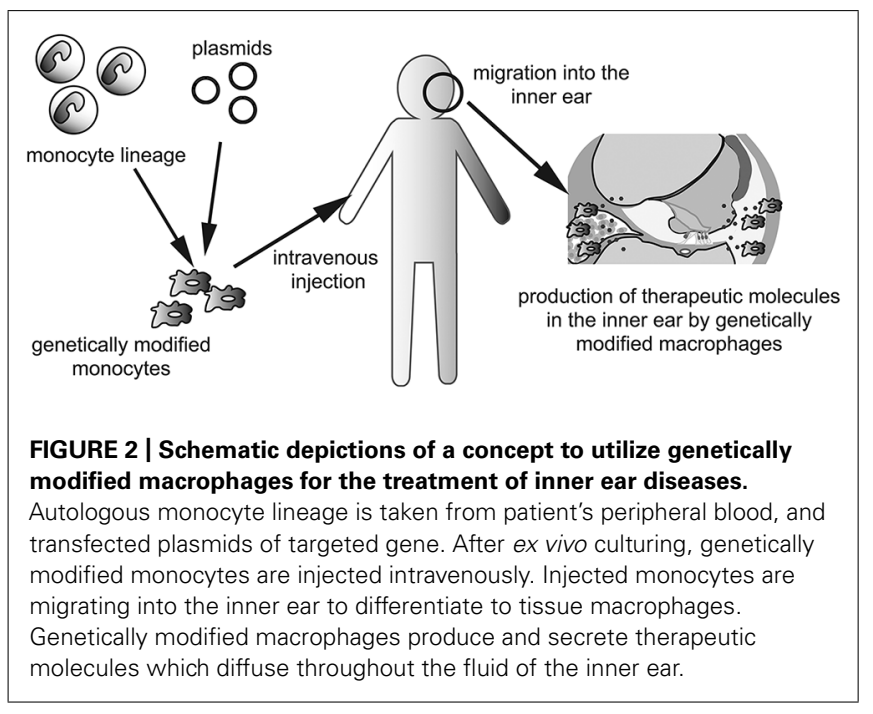

cytokines, and cell adhesion molecules. An alternative approach is to facilitate phagocytosis of loaded delivery vehicles by monocytes, which then passively targets the site of disease due to the mounting immune response. The active targeting approach is most attractive and promising if the surface of the delivery vehicle can be decorated with a ligand that selectively interacts with their target receptors. Further research evaluating the use of monocytes as vehicles is desired.

\section{CONCLUSION}

In this review, we discussed the involvement of the immune system in the pathology of SNHL, particularly the innate immune system in the inner ear and the pathology of immune-mediated inner ear disease. Recent advances in basic and clinical audiology and immunology research has been rapid. Although there is still much work to be done, we believe that the future of inner ear immunology and SNHL treatment are bright and promising.

\section{ACKNOWLEDGMENTS}

This study was supported by funds from the Shimizu Foundation of Immunology and Neuroscience Grant for 2012 and a Grant-in-Aid for Scientific Research from the Ministry of Education, Culture, Sports, Science and Technology in Japan and Japan Society for Promotion of Science.

\section{REFERENCES}

Alexander, T. H., Weisman, M. H., Derebery, J. M., Espeland, M. A., Gantz, B. J., Gulya, A. J., et al. (2009). Safety of high-dose corticosteroids for the treatment of autoimmune inner ear disease. Otol. Neurotol. 30, 443-448. doi: 10.1097/MAO.0b013e3181a52773

Alleman, A. M., Dornhoffer, J. L., Arenberg, I. K., and Walker, P. D. (1997). Demonstration of autoantibodies to the endolymphatic sac in Meniere's disease. Laryngoscope 107, 211-215. doi: 10.1097/00005537-199702000-00013

Arvin, A. M., Fast, P., Myers, M., Plotkin, S., and Rabinovich, R. (2004). Vaccine development to prevent cytomegalovirus disease: report from the national vaccine advisory committee. Clin. Infect. Dis. 39, 233-239. doi: 10.1086/421999

Assuiti, L. F., Lanzoni, G. M., Santos, F. C., Erdmann, A. L., and Meirelles, B. H. (2013). Hearing loss in people with HIV/AIDS, and associated factors: an integrative review. Braz. J. Otorhinolaryngol. 79, 248-255. doi: 10.5935/18088694.20130042 
Barbi, M., Binda, S., Caroppo, S., Ambrosetti, U., Corbetta, C., and Sergi, P. (2003). A wider role for congenital cytomegalovirus infection in sensorineural hearing loss. Pediatr. Infect. Dis. J. 22, 39-42. doi: 10.1097/01.inf.0000047673.38917.e4

Bergstrom, T., Edstrom, S., Tjellstrom, A., and Vahlne, A. (1992). Meniere's disease, and antibody reactivity to herpes simplex virus type 1 polypeptides. Am. J. Otolaryngol. 13, 295-300. doi: 10.1016/0196-0709(92) 90051-T

Bhave, S. A., Oesterle, E. C., and Coltrera, M. D. (1998). Macrophage, and microglia-like cells in the avian inner ear. J. Comp. Neurol. 398, 241-256. doi: 10.1002/(SICI) 1096-9861(19980824)398:2<241::AID-CNE6>3.0.CO;2-0

Burdo, T. H., Lackner, A., and Williams, K. C. (2013). Monocyte/macrophages, and their role in HIV neuropathogenesis. Immunol. Rev. 254, 102-113. doi: 10.1111/imr.12068

Cohen, S., Roland, P., Shoup, A., Lowenstein, M., Silverstein, H., Kavanaugh, A., et al. (2011). A pilot study of rituximab in immune-mediated inner ear disease Audiol. Neurootol. 16, 214-221. doi: 10.1159/000320606

Cohen, S., Shoup, A., Weisman, M. H., and Harris, J. (2005). Etanercept treatment for autoimmune inner ear disease: results of a pilot placebo-controlled study. Otol. Neurotol. 26, 903-907. doi: 10.1097/01.mao.0000185082.28598.87

Derebery, M. J., Rao, V. S., Siglock, T. J., Linthicum, F. H., and Nelson, R. A. (1991). Meniere's disease: an immune complex-mediated illness? Laryngoscope 101, 225-229. doi: 10.1288/00005537-199103000-00001

Dettmer, M., Hegemann, I., and Hegemann, S. C. (2011). Extraintestinal Crohn's disease mimicking autoimmune inner ear disease: a histopathological approach. Audiol. Neurootol. 16, 36-40. doi: 10.1159/000315063

Dornhoffer, J. L., Waner, M., Arenberg, I. K., and Montague, D. (1993). Immunoperoxidase study of the endolymphatic sac in Meniere's disease. Laryngoscope 103, 1027-1034. doi: 10.1288/00005537-199309000-00014

Garcia-Berrocal, J. R., Ibanez, A., Rodriguez, A., Gonzalez-Garcia, J. A., Verdaguer, J. M., Trinidad, A., et al. (2006). Alternatives to systemic steroid therapy for refractory immune-mediated inner ear disease: a physiopathologic approach. Eur. Arch. Otorhinolaryngol. 263, 977-982. doi: 10.1007/s00405-0060096-9

Garcia-Berrocal, J. R., Vargas, J. A., Ramirez-Camacho, R. A., Gonzalez, F. M., GeaBanacloche, J. C., Vergara, J., et al. (1997). Deficiency of naive T cells in patients with sudden deafness. Arch. Otolaryngol. Head Neck Surg. 123, 712-717. doi: 10.1001/archotol.1997.01900070056009

Hakuba, N., Hata, R., Morizane, I., Feng, G., Shimizu, Y., Fujita, K., et al. (2005). Neural stem cells suppress the hearing threshold shift caused by cochlear ischemia. Neuroreport 16, 1545-1549.

Han, C. S., Park, J. R., Boo, S. H., Jo, J. M., Park, K. W., Lee, W. Y., et al. (2009). Clinical efficacy of initial intratympanic steroid treatment on sudden sensorineural hearing loss with diabetes. Otolaryngol. Head Neck Surg. 141, 572-578. doi: 10.1016/j.otohns.2009.06.084

Harris, J. P., Heydt, J., Keithley, E. M., and Chen, M. C. (1997). Immunopathology of the inner ear: an update. Ann. N. Y. Acad. Sci. 830, 166-178. doi: 10.1111/j.17496632.1997.tb51888.x

Harris, J. P., and Ryan, A. F. (1984). Immunobiology of the inner ear. Am. J. Otolaryngol. 5, 418-425. doi: 10.1016/S0196-0709(84)80059-9

Harris, J. P., Weisman, M. H., Derebery, J. M., Espeland, M. A., Gantz, B. J., Gulya, A. J., et al. (2003). Treatment of corticosteroid-responsive autoimmune inner ear disease with methotrexate: a randomized controlled trial. JAMA 290, 1875-1883. doi: 10.1001/jama.290.14.1875

Hirose, K., Discolo, C. M., Keasler, J. R., and Ransohoff, R. (2005). Mononuclear phagocytes migrate into the murine cochlea after acoustic trauma. J. Comp. Neurol. 489, 180-194. doi: 10.1002/cne.20619

Hughes, G. B., Kinney, S. E., Barna, B. P., and Calabrese, L. H. (1983). Autoimmune reactivity in Meniere's disease: a preliminary report. Laryngoscope 93, 410-417.

Hume, D. A., Ross, I. L., Himes, S. R., Sasmono, R. T., Wells, C. A., and Ravasi, T. (2002). The mononuclear phagocyte system revisited. J. Leukoc. Biol. 72, 621-627.

Imai, Y., Ibata, I., Ito, D., Ohsawa, K., and Kohsaka, S. (1996). A novel gene ibal in the major histocompatibility complex class III region encoding an EF hand protein expressed in a monocytic lineage. Biochem. Biophys. Res. Commun. 224, 855-862. doi: 10.1006/bbrc.1996.1112

Jabba, S. V., Oelke, A., Singh, R., Maganti, R. J., Fleming, S., Wall, S. M., et al. (2006). Macrophage invasion contributes to degeneration of stria vascularis in Pendred syndrome mouse model. BMC Med. 4:37. doi: 10.1186/17417015-4-37
Juanjuan, C., Yan, F., Li, C., Haizhi, L., Ling, W., Xinrong, W., et al. (2011). Murine model for congenital CMV infection, and hearing impairment. Virol. J. 8:70. doi: 10.1186/1743-422X-8-70

Kakehata, S., Sasaki, A., Oji, K., Futai, K., Ota, S., Makinae, K., et al. (2006). Comparison of intratympanic, and intravenous dexamethasone treatment on sudden sensorineural hearing loss with diabetes. Otol. Neurotol. 27, 604-608. doi: 10.1097/01.mao.0000224092.79635.ee

Kesser, B. W., and Lalwani, A. K. (2009). Gene therapy, and stem cell transplantation: strategies for hearing restoration. Adv. Otorhinolaryngol. 66, 64-86. doi: $10.1159 / 000218208$

Kumagami, H. (1996). Detection of viral antigen in the endolymphatic sac. Eur. Arch. Otorhinolaryngol. 253, 264-267. doi: 10.1007/BF00171140

Lang, H., Ebihara, Y., Schmiedt, R. A., Minamiguchi, H., Zhou, D., Smythe, N., et al. (2006). Contribution of bone marrow hematopoietic stem cells to adult mouse inner ear: mesenchymal cells, and fibrocytes. J. Comp. Neurol. 496, 187-201. doi: $10.1002 /$ cne.20929

Lasak, J. M., Sataloff, R. T., Hawkshaw, M., Carey, T. E., Lyons, K. M., and Spiegel, J. R. (2001). Autoimmune inner ear disease: steroid, and cytotoxic drug therapy. Ear Nose Throat J. 80, 808-811, 815-816, 818 passim.

Lawrence, T., and Natoli, G. (2011). Transcriptional regulation of macrophage polarization: enabling diversity with identity. Nat. Rev. Immunol. 11, 750-761. doi: 10.1038/nri3088

Lin, C., Lin, S. W., Weng, S. F., and Lin, Y. S. (2013). Increased risk of sudden sensorineural hearing loss in patients with human immunodeficiency virus aged 18 to 35 years: a population-based cohort study. JAMA Otolaryngol. Head Neck Surg. 139, 251-255. doi: 10.1001/jamaoto.2013.1709

Linthicum, F. H. Jr., Doherty, J., and Berliner, K. I. (2013). Idiopathic sudden sensorineural hearing loss: vascular or viral? Otolaryngol. Head Neck Surg. 149, 914-917. doi: 10.1177/0194599813506546

Liu, Y. C., Rubin, R., and Sataloff, R. T. (2011). Treatment-refractory autoimmune sensorineural hearing loss: response to infliximab. Ear Nose Throat J. 90, 23-28.

Lu, M. H., Takemoto, M., Watanabe, K., Luo, H., Nishimura, M., Yano, M., et al. (2012). Deficiency of sphingomyelin synthase-1 but not sphingomyelin synthase2 causes hearing impairments in mice. J. Physiol. 590(Pt 16), 4029-4044. doi: 10.1113/jphysiol.2012.235846

MacArthur, C. J., Kempton, J. B., DeGagne, J., and Trune, D. R. (2008). Control of chronic otitis media, and sensorineural hearing loss in $\mathrm{C} 3 \mathrm{H} / \mathrm{HeJ}$ mice: glucocorticoids vs mineralocorticoids. Otolaryngol. Head Neck Surg. 139, 646-653. doi: 10.1016/j.otohns.2008.07.029

Mark, A. S., Seltzer, S., Nelson-Drake, J., Chapman, J. C., Fitzgerald, D. C., and Gulya, A. J. (1992). Labyrinthine enhancement on gadolinium-enhanced magnetic resonance imaging in sudden deafness, and vertigo: correlation with audiologic, and electronystagmographic studies. Ann. Otol. Rhinol. Laryngol. 101, 459-464.

Matteson, E. L., Choi, H. K., Poe, D. S., Wise, C., Lowe, V. J., McDonald, T. J., et al. (2005). Etanercept therapy for immune-mediated cochleovestibular disorders: a multi-center, open-label, pilot study. Arthritis Rheum. 53, 337-342. doi: 10.1002/art.21179

Matteson, E. L., Fabry, D. A., Facer, G. W., Beatty, C. W., Driscoll, C. L., Strome, S. E., etal. (2001). Open trial of methotrexate as treatment for autoimmune hearing loss. Arthritis Rheum. 45, 146-150. doi: 10.1002/15290131(200104)45:2<146::AID-ANR167>3.0.CO;2-I

McCabe, B. F. (1979). Autoimmune sensorineural hearing loss. Ann. Otol. Rhinol. Laryngol. 88(5Pt 1), 585-589.

McCabe, B. F. (1989). Autoimmune inner ear disease: therapy. Am. J. Otol. 10, 196-197.

Meltser, I., Tahera, Y., and Canlon, B. (2009). Glucocorticoid receptor, and mitogenactivated protein kinase activity after restraint stress, and acoustic trauma. $J$. Neurotrauma 26, 1835-1845. doi: 10.1089/neu.2008.0874

Merchant, S. N., Durand, M. L., and Adams, J. C. (2008). Sudden deafness: is it viral? ORL J. Otorhinolaryngol. Relat. Spec. 70, 52-60; discussion 60-62. doi: $10.1159 / 000111048$

Mohler, K. M., Torrance, D. S., Smith, C. A., Goodwin, R. G., Stremler, K. E., Fung, V. P., et al. (1993). Soluble tumor necrosis factor (TNF) receptors are effective therapeutic agents.in lethal endotoxemia and function simultaneously as both TNF carriers and TNF antagonists. J. Immunol. 151, $1548-1561$. 
Morovic Vergles, J., Radic, M., Kovacic, J., and Salamon, L. (2010). Successful use of adalimumab for treating rheumatoid arthritis with autoimmune sensorineural hearing loss: two birds with one stone. J. Rheumatol. 37, 1080-1081. doi: 10.3899/jrheum.091342

Moscicki, R. A., San Martin, J. E., Quintero, C. H., Rauch, S. D., Nadol, J. B. Jr., and Bloch, K. J. (1994). Serum antibody to inner ear proteins in patients with progressive hearing loss. correlation with disease activity, and response to corticosteroid treatment. JAMA 272, 611-616. doi: 10.1001/jama.1994.0352008 0053043

Numazaki, K., and Fujikawa, T. (2004). Chronological changes of incidence, and prognosis of children with asymptomatic congenital cytomegalovirus infection in Sapporo, Japan. BMC Infect. Dis. 4:22. doi: 10.1186/1471-2334-4-22

Okano, T., Nakagawa, T., Kita, T., Endo, T., and Ito, J. (2006). Cell-gene delivery of brain-derived neurotrophic factor to the mouse inner ear. Mol. Ther. 14, 866-871. doi: 10.1016/j.ymthe.2006.06.012

Okano, T., Nakagawa, T., Kita, T., Kada, S., Yoshimoto, M., Nakahata, T., et al. (2008) Bone marrow-derived cells expressing Ibal are constitutively present as resident tissue macrophages in the mouse cochlea. J. Neurosci. Res. 86, 1758-1767. doi: 10.1002/jnr.21625

Pyykko, I., and Zou, J. (2008). Do viruses cause inner ear disturbances? ORL J. Otorhinolaryngol. Relat. Spec. 70, 32-40; discussion 40-41. doi: $10.1159 / 000111046$

Rahman, M. U., Poe, D. S., and Choi, H. K. (2001). Etanercept therapy for immune-mediated cochleovestibular disorders: preliminary results in a pilot study. Otol. Neurotol. 22, 619-624. doi: 10.1097/00129492-20010900000010

Ramprasad, M. P., Terpstra, V., Kondratenko, N., Quehenberger, O., and Steinberg, D. (1996). Cell surface expression of mouse macrosialin, and human CD68, and their role as macrophage receptors for oxidized low density lipoprotein. Proc. Natl. Acad. Sci. U.S.A. 93, 14833-14838. doi: 10.1073/pnas.93.25. 14833

Rask-Andersen, H., and Stahle, J. (1979). Lymphocyte-macrophage activity in the endolymphatic sac. An ultrastructural study of the rugose endolymphatic sac in the guinea pig. ORL J. Otorhinolaryngol. Relat. Spec. 41, 177-192. doi: $10.1159 / 000275458$

Salley, L. H. Jr., Grimm, M., Sismanis, A., Spencer, R. F., and Wise, C. M. (2001). Methotrexate in the management of immune mediated cochleovesitibular disorders: clinical experience with 53 patients. J. Rheumatol. 28 , 1037-1040.

Sato, E., Shick, H. E., Ransohoff, R. M., and Hirose, K. (2008). Repopulation of cochlear macrophages in murine hematopoietic progenitor cell chimeras: the role of CX3CR1. J. Comp. Neurol. 506, 930-942. doi: 10.1002/cne. 21583

Sato, E., Shick, H. E., Ransohoff, R. M., and Hirose, K. (2010). Expression of fractalkine receptor CX3CR1 on cochlear macrophages influences survival of hair cells following ototoxic injury. J. Assoc. Res. Otolaryngol. 11, 223-234. doi 10.1007/s10162-009-0198-3

Siddiqui, M. A., and Scott, L. J. (2005). Infliximab: a review of its use in Crohn's disease, and rheumatoid arthritis. Drugs 65, 2179-2208. doi: 10.2165/00003495200565150-00014

Singh, R., and Wangemann, P. (2008). Free radical stress-mediated loss of Kcnj10 protein expression in stria vascularis contributes to deafness in Pendred syndrome mouse model. Am. J. Physiol. Renal Physiol. 294, F139-F148. doi: 10.1152/ajprenal.00433.2007

Smith, M. J., and Koch, G. L. (1987). Differential expression of murine macrophage surface glycoprotein antigens in intracellular membranes. J. Cell Sci. 87(Pt 1), 113-119.

Spear, S. A., and Schwartz, S. R. (2011). Intratympanic steroids for sudden sensorineural hearing loss: a systematic review. Otolaryngol. Head Neck Surg. 145 534-543. doi: 10.1177/0194599811419466

Stokroos, R. J., Albers, F. W., Krikke, A. P., and Casselman, J. W. (1998). Magnetic resonance imaging of the inner ear in patients with idiopathic sudden sensorineural hearing loss. Eur. Arch. Otorhinolaryngol. 255, 433-436. doi: $10.1007 /$ s004050050093
Tahera, Y., Meltser, I., Johansson, P., Bian, Z., Stierna, P., Hansson, A. C., et al. (2006). NF-kappaB mediated glucocorticoid response in the inner ear after acoustic trauma. J. Neurosci. Res. 83, 1066-1076. doi: 10.1002/jnr.20795

Tebo, A. E., Szankasi, P., Hillman, T. A., Litwin, C. M., and Hill, H. R. (2006). Antibody reactivity to heat shock protein 70 , and inner ear-specific proteins in patients with idiopathic sensorineural hearing loss. Clin. Exp. Immunol. 146, 427-432. doi: 10.1111/j.1365-2249.2006.03227.x

Tornabene, S. V., Sato, K., Pham, L., Billings, P., and Keithley, E. M. (2006) Immune cell recruitment following acoustic trauma. Hear. Res. 222, 115-124. doi: 10.1016/j.heares.2006.09.004

Trune, D. R., and Canlon, B. (2012). Corticosteroid therapy for hearing, and balance disorders. Anat. Rec. (Hoboken) 295, 1928-1943. doi: 10.1002/ ar.22576

Van Wijk, F., Staecker, H., Keithley, E., and Lefebvre, P. P. (2006). Local perfusion of the tumor necrosis factor alpha blocker infliximab to the inner ear improves autoimmune neurosensory hearing loss. Audiol. Neurootol. 11, 357-365. doi: $10.1159 / 000095897$

Vincent, V. A., Robinson, C. C., Simsek, D., and Murphy, G. M. (2002). Macrophage colony stimulating factor prevents NMDA-induced neuronal death in hippocampal organotypic cultures. J. Neurochem. 82, 1388-1397. doi: 10.1046/j.1471-4159.2002.01087.x

Wang, Y., Patel, R., Ren, C., Taggart, M. G., Firpo, M. A., Schleiss, M. R., et al. (2013). A comparison of different murine models for cytomegalovirus-induced sensorineural hearing loss. Laryngoscope 123, 2801-2806. doi: 10.1002/lary. 24090

Warchol, M. E. (1997). Macrophage activity in organ cultures of the avian cochlea: demonstration of a resident population, and recruitment to sites of hair cell lesions. J. Neurobiol. 33, 724-734. doi: 10.1002/(SICI)10974695(19971120)33:6<724::AID-NEU2>3.0.CO;2-B

Woolf, N. K., Koehrn, F. J., Harris, J. P., and Richman, D. D. (1989) Congenital cytomegalovirus labyrinthitis, and sensorineural hearing loss in guinea pigs. J. Infect. Dis. 160, 929-937. doi: 10.1093/infdis/160. 6.929

Wynn, T. A., Chawla, A., and Pollard, J. W. (2013). Macrophage biology in development, homeostasis, and disease. Nature 496, 445-455. doi: 10.1038/ nature 12034

Yagihashi, A., Sekiya, T., and Suzuki, S. (2005). Macrophage colony stimulating factor (M-CSF) protects spiral ganglion neurons following auditory nerve injury: morphological, and functional evidence. Exp. Neurol. 192, 167-177. doi: 10.1016/j.expneurol.2004.10.020

Zeitoun, H., Beckman, J. G., Arts, H. A., Lansford, C. D., Lee, D. S., El-Kashlan, H. K., et al. (2005). Corticosteroid response, and supporting cell antibody in autoimmune hearing loss. Arch. Otolaryngol. Head Neck Surg. 131, 665-672. doi: 10.1001/archotol.131.8.665

Zhang, W., Dai, M., Fridberger, A., Hassan, A., Degagne, J., Neng, L., et al. (2012). Perivascular-resident macrophage-like melanocytes in the inner ear are essential for the integrity of the intrastrial fluid-blood barrier. Proc. Natl. Acad. Sci. U.S.A. 109, 10388-10393. doi: 10.1073/pnas.1205210109

Conflict of Interest Statement: The author declares that the research was conducted in the absence of any commercial or financial relationships that could be construed as a potential conflict of interest.

Received: 30 July 2014; accepted: 16 August 2014; published online: 02 September 2014. Citation: Okano T (2014) Immune system of the inner ear as a novel therapeutic target for sensorineural hearing loss. Front. Pharmacol. 5:205. doi: 10.3389/fphar.2014.00205 This article was submitted to Pharmaceutical Medicine and Outcomes Research, a section of the journal Frontiers in Pharmacology.

Copyright $(2014$ Okano. This is an open-access article distributed under the terms of the Creative Commons Attribution License (CC BY). The use, distribution or reproduction in other forums is permitted, provided the original author(s) or licensor are credited and that the original publication in this journal is cited, in accordance with accepted academic practice. No use, distribution or reproduction is permitted which does not comply with these terms. 